\title{
Where stars form and live at high redshift: clues from the infrared
}

\author{
M. Béthermin ${ }^{1,2}$, O. Doré 3,4 , and G. Lagache ${ }^{2}$ \\ 1 Laboratoire AIM-Paris-Saclay, CEA/DSM/Irfu, CNRS, Univ. Paris Diderot, Saclay, France \\ e-mail: matthieu. bethermin@cea.fr \\ 2 Institut d'Astrophysique Spatiale (IAS), Bat. 121, 91405 Orsay, France; Université Paris-Sud 11 and CNRS (UMR8617), France \\ 3 Jet Propulsion Laboratory, California Institute of Technology, 4800 Oak Grove Drive, Pasadena, California, USA \\ 4 California Institute of Technology, Pasadena, California, USA
}

Received 7 December 2011 / Accepted 24 December 2011

\section{ABSTRACT}

\begin{abstract}
The relation between dark matter halos and the loci of star formation at high redshift is a pressing question in contemporary cosmology. Matching the abundance of halos to the abundance of infrared (IR) galaxies, we explore the link between dark matter halo mass ( $M_{\mathrm{h}}$ ), stellar mass $\left(M_{\star}\right)$ and star-formation rate (SFR) up to a redshift of 2. Our findings are five-fold. First, we find a strong evolution of the relation between $M_{\star}$ and SFR as a function of redshift with an increase of $\mathrm{sSFR}=\mathrm{SFR} / M_{\star}$ by a factor $\sim 30$ between $z=0$ and $z=2.3$. Second, we observe a decrease of sSFR with stellar mass. These results reproduce observed trends at redshift $z>0.3$. Third, we find that the star formation is most efficient in dark matter halos with $M_{\mathrm{h}} \simeq 5 \times 10^{11} M_{\odot}$, with hints of an increase of this mass with redshift. Fourth, we find that SFR $/ M_{\mathrm{h}}$ increases by a factor $\sim 15$ between $z=0$ and $z=2.3$. Finally we find that the SFR density is dominated by halo masses close to $\sim 7 \times 10^{11} M_{\odot}$ at all redshift, with a rapid decrease at lower and higher halo masses. Despite its simplicity, our novel use of IR observations unveils some characteristic mass-scales governing star formation at high redshift.
\end{abstract}

Key words. galaxies: star formation - galaxies: statistics - galaxies: halos - dark matter - infrared: galaxies

\section{Introduction}

The loci of star formation at high redshift is one of the salient questions of galaxy formation theory. In particular, uncertainties in the relation between the host halo mass and the IR luminosity function (LFIR) currently limit models of the clustering of star-forming galaxies as revealed e.g. by the anisotropies of the cosmic infrared background (CIB). This comes from the fact that the clustering of galaxies is directly driven by the mass of their host halos. The so-called abundance matching technique is another valuable tool that provides insights on this relation (Vale \& Ostriker 2004). While unexplored in the IR, a recent improvement of LFIR measurements makes this approach now both promising and timely. We will illustrate its power in this letter. In particular, working in the IR provides the unique possibility to constrain the star-formation rate (SFR) directly without any extra assumption regarding the physical processes that drive the star formation.

Considering the latest observational and theoretical developments in the IR, we introduce in Sect. 2 the abundance matching technique. We describe in detail the mass and luminosity functions used in Sect. 3. Matching abundances allows us to infer relations between halo and stellar mass (Sect. 4.1), SFR and stellar mass (Sect. 4.2) and SFR and halo mass (Sect. 4.3). In these sections, we will illustrate in particular how this simple but well-informed approach satisfyingly reproduces trends observed in the IR, but also at other wavelengths. Focusing on what is required for the modeling of the CIB, we exclude the so-called starbursting galaxies here because they are non-significant outliers. Critical in the context of abundance matching, this assumption is well motivated and discussed in Sect. 3.
Throughout this paper, we adopt a Chabrier (2003) initial mass function (IMF) ${ }^{1}$ and a $\Lambda$ CDM WMAP-7 cosmology (Larson et al. 2011).

\section{Simple abundance matching}

Our goal is to find an observationally supported relation between the SFR of galaxies, their stellar and their host halo mass. While this relation is statistical in nature, the abundance matching technique (e.g. Conroy \& Wechsler 2009) provides a way to relate the means of this relation. Observations suggest a small scatter around this relation and we will therefore neglect it: 0.16 dex for the relationship between stellar and halo mass (More et al. 2009), and 0.2 dex between the stellar mass and the SFR (Rodighiero et al. 2011; see also Behroozi et al. 2010 for a more general discussion of scatter).

The key assumption of this matching technique is the existence of a continuous and monotonic relation between the halo mass, the stellar mass, and the SFR: SFR $=f\left(M_{\star}\right), \mathrm{SFR}=g\left(M_{\mathrm{h}}\right)$ and $M_{\star}=h\left(M_{\mathrm{h}}\right)$, where $S F R$ is the SFR, $M_{\star}$ the galaxy stellar mass (SM), and $M_{\mathrm{h}}$ the dark-matter halo mass (HM).

We solve for the functions $f, g$ and $h$ that satisfy the following relations between the infrared luminosity function, the halo mass function, and the stellar mass function:

$n_{L_{\mathrm{IR}}}\left(>L_{\mathrm{IR}}=K^{-1} \times f\left(M_{\star}\right)\right)=n_{M_{\star}}\left(>M_{\star}\right)$

$n_{L_{\mathrm{IR}}}\left(>L_{\mathrm{IR}}=K^{-1} \times g\left(M_{\mathrm{h}}\right)\right)=n_{M_{\mathrm{h}}}\left(>M_{\mathrm{h}}\right)$

$$
n_{M_{\star}}\left(>M_{\star}=h\left(M_{\mathrm{h}}\right)\right)=n_{M_{\mathrm{h}}}\left(>M_{\mathrm{h}}\right),
$$

1 Stellar masses and SFR computed assuming a Salpeter (1955) IMF can be converted to Chabrier (2003) IMF applying a -0.24 dex correction. 
where $n_{L_{\mathrm{IR}}}$ is the number of galaxies per unit comoving volume brighter than the bolometric infrared luminosity $L_{\mathrm{IR}}$, and $n_{M_{\star}}$ and $n_{M_{\mathrm{h}}}$ are the number of galaxies more massive than the stellar mass $M_{\star}$ and the halo mass $M_{\mathrm{h}}$, respectively ${ }^{2} . K$ is the Kennicutt (1998) factor, which links the infrared bolometric luminosity integrated between 8 and $1000 \mu \mathrm{m}$ and the SFR: $K=10^{-10} M_{\odot} \mathrm{yr}^{-1} L_{\odot}^{-13}$. As we discuss below, a key assumption in this matching is that all (sub)halos contribute to the IR.

\section{Mass and luminosity functions}

The key inputs to our discussions are the bolometric infrared luminosity function (LFIR), the stellar mass function (SMF) and the halo mass function (HMF).

It is well known that the LFIR is inconsistent with the Schechter (1976) form and presents an excess at the bright end (e.g. Saunders et al. 1990). However, it has been shown that this excess is mainly caused by starbursting galaxies, which are well outside the SFR-SM main sequence. Yun et al. (2001) showed that the $60 \mu \mathrm{m}$ LF of the IRAS 2 Jy sample can be fitted by the sum of two Schechter functions. More recently, Sargent et al. (2011) showed that the LFIR can be decomposed as a sum of two approximate Schechter functions. The first one is responsible for $\sim 85 \%$ of the IR energy output, and is caused by the so-called main-sequence objects that have very similar specific sSFR at a given $M_{\star}$ and redshift. The second one dominates the bright-end of the LF, and is caused by starbursting galaxies with an SSSFR about 0.5 dex higher than the main sequence. These starbursts contribute only $\sim 2 \%$ to the mass-selected star-forming galaxies number density (Rodighiero et al. 2011), and $\sim 15 \%$ to the SFR density (Sargent et al. 2011), and consequently to the CIB. The former guarantees that starbursts constitute a negligible fraction of IR objects, i.e. do not contribute significantly to the IR abundances. The latter suggests that they can be neglected for our purpose. Additionally, because a bimodal relation between LFIR and SSFR would violate our hypothesis of a monotonic relation between masses and SFRs, we henceforth ignore the starburst components. Focusing on the main-sequence population, we therefore write the LFIR as a Schechter (1976) function

$\frac{\mathrm{d} N}{\mathrm{~d} L_{\mathrm{IR}}}=\phi_{\star}(z)\left(\frac{L_{\mathrm{IR}}}{L_{\star}(z)}\right)^{-\alpha} \exp \left(\frac{L_{\mathrm{IR}}}{L_{\star}(z)}\right) \frac{1}{L_{\star}(z)}$,

where $\mathrm{d} N / \mathrm{d} L_{\mathrm{IR}}$ is the LFIR, and $\phi_{\star}(z)$ and $L_{\star}(z)$ are the characteristic density and luminosity at a given redshift, respectively. We use the evolution of $\phi_{\star}(z)$ and $L_{\star}(z)$ measured by Magnelli et al. (2011) up to $z=2.3$.

In contrast to the LFIR, the SMF is much closer to a Schechter (1976) function. Pérez-González et al. (2008) have measured the SMF up to $z=4$ and we used their parameters of the Schechter function fit. We performed our abundance matching at the same redshift as the center of the redshift bins of Pérez-González et al. (2008). We checked that there is no inconsistency between the SFR derived from our LFIR and that derived from the SMF following Wilkins et al. (2008).

For the HMF, we used the Tinker et al. (2008) z-dependent $\mathrm{HMF}$ and the associated sub-halo mass function proposed in Tinker \& Wetzel (2010) with $\Delta=200$. Our results are fairly insensitive to the inclusion of the sub-halo mass function.

\footnotetext{
${ }^{2}$ Note that $n$ can be deduced from the luminosity/mass function: $n_{X}\left(>X_{\min }\right)=\int_{X_{\min }}^{+\infty} \frac{\mathrm{d}^{2} N}{\mathrm{~d} X \mathrm{~d} V} \mathrm{~d} X$, where $X$ is $L_{\mathrm{IR}}, M_{\star}$, or $M_{\mathrm{h}}$.

3 The Kennicutt factor was re-scaled from the Salpeter to the Chabrier IMF.
}

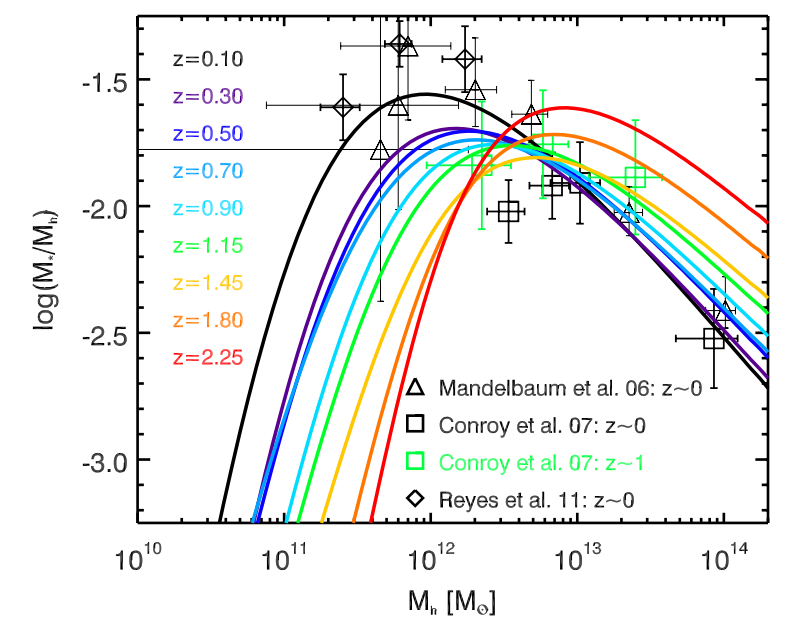

Fig. 1. Ratio between stellar and halo mass as a function of halo mass from $z=0$ to $z=2.25$. Solid lines: our results based on abundance matching technique. Triangles: measurements from galaxygalaxy lensing (Mandelbaum et al. 2006). Squares: measurements from satellite galaxies (Conroy et al. 2007) at $z \sim 0$ (black) and $z \sim 1$ (green). Diamonds: measurements from combined kinematics and galaxy-galaxy lensing (Reyes et al. 2011). Typical uncertainties on the results of abundance matching are 0.2 dex.

\section{Results}

\subsection{Relation between stellar mass and halo mass}

The abundance matching between the HMF and SMF has been extensively studied for $z<1$ (e.g. Conroy \& Wechsler 2009) and has been discussed in detail up to $z \sim 4$ by Behroozi et al. (2010). Because $M_{\star} / M_{\mathrm{h}}$ as a function of $M_{\mathrm{h}}$ is one basic element of our analysis, we first investigated it using our mass functions. Figure 1 shows this relation relation up to $z \sim 2.25$. It agrees with the measurements at $z \sim 0$ and $z \sim 1$ (Mandelbaum et al. 2006; Conroy et al. 2007; Reyes et al. 2011). However, constraints are poor except at $z=0$ for $M_{\mathrm{h}}>10^{11} M_{\odot}$. We find that $M_{\star} / M_{\mathrm{h}}$ peaks near $M_{\mathrm{h}}=10^{12} M_{\odot}$ and decreases at lower- and highermass. This suggests that the integrated star-formation efficiency $\left(\equiv M_{\star} / M_{\mathrm{h}}\right)$ decreases for smaller as well as higher-mass halos. Figure 1 also indicates that the mass corresponding to the peak of integrated star-formation efficiency evolves with redshift, being a factor of 8 larger at $z=2.25$ than at $z=0$. We extended our analysis to higher redshifts using the latest SMF measurements (Caputi et al. 2011; Kajisawa et al. 2009) and confirm this trend. However, as discussed in Behroozi et al. (2010), these results have to be taken with care because the current uncertainties make these evolutions quite uncertain.

\subsection{Relation between star-formation rate and stellar mass}

Matching the abundances derived from the LFIR and the SMF, we plot in Fig. 2 the sSFR as a function of $M_{\star}$ and redshift. We observe a strong increase of the sSFR with redshift (about 1.5 order of magnitude between $z=0$ and $z=2$ ), which qualitatively agrees with observations (Elbaz et al. 2011; Karim et al. 2011). We also see a slight decrease of the sSFR with $M_{\star}(0.5 \mathrm{dex}$ between $M_{\star}=10^{9.5} M_{\odot}$ and $\left.M_{\star}=10^{11.5} M_{\odot}\right)$, which again agrees with the observations (Rodighiero et al. 2011; Karim et al. 2011). To further illustrate this qualitative agreement, we also plot in Fig. 2 the most recent sSFR measurements. We reproduce their trends quite well. We note the large dispersion between the measurements, however. For example, Karim et al. (2011) and Rodighiero et al. (2011) differ significantly at high-mass 
M. Béthermin et al.: Where stars form and live at high redshift: clues from the infrared

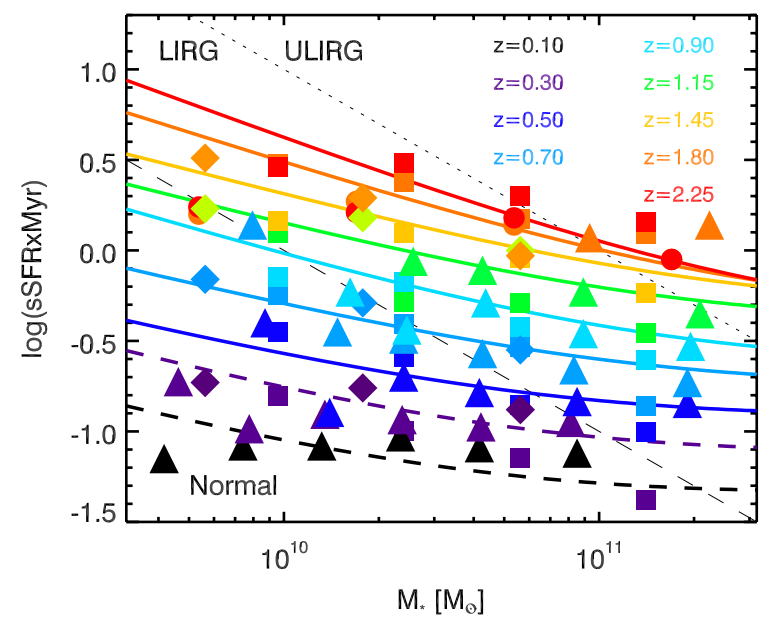

Fig. 2. sSFR as a function of stellar mass at various redshifts. Solid lines: our results based on the abundance matching technique (we use dashed lines at $z \leq 0.3$ where neglecting the quiescent galaxies have a significant impact, see Sect. 5). Filled squares: measurements from Karim et al. (2011) obtained by radio stacking at $z=0.3,0.5,0.7,0.9$, $1.1,1.4,1.8$, and 2.25. Filled diamonds: measurement from Rodighiero et al. (2010) obtained by Herschel/PACS stacking at $z=0.25,0.75$, 1.25, and 1.75. Filled circles: measurements from Bauer et al. (2011) obtained using UV measurements at $z=1.75$ and 2.25. Filled triangles: measurements of Oliver et al. (2010) from Spitzer/MIPS stacking at $z=0.1,0.3,0.5,0.7,0.9,1.1,1.35,1.75$. The error bars have not been plotted for clarity, but they roughly match the symbol size. Thin-dashed and dotted lines are the limits between the normal and LIRG (luminous infrared galaxies, $10^{11} L_{\odot}<L_{\mathrm{IR}}<10^{12} L_{\odot}$ ), and the LIRG and ULIRG (ultra luminous infrared galaxies, $L_{\mathrm{IR}}>10^{12} L_{\odot}$ ) regime, respectively.

at $z \sim 0.3$, and at all masses for $z \sim 1$ (note however that the redshift bins are quite different). Also, at higher redshift the measurements seem to differ by factors $\sim 2$ around $10^{10} M_{\odot}$.

\subsection{Relation between star-formation rate and halo mass}

We have seen in Sects. 4.1 and 4.2 that matching abundances leads to elucidating connections between the stellar and halo mass on the one hand, and the stellar mass and the sSFR on the other hand. It is therefore natural to investigate the connection between the halo mass and the SFR. We can make this connection in two ways. In Fig. 3 (upper panel), we plot the redshift evolution of the ratio between SFR and halo mass as a function of halo mass. The solid lines use a direct matching between the LFIR and the HMF. The dotted-dashed lines stem from a combination between the function linking the stellar mass and the halo mass, and the power-law fit of the sSFR- $M_{\star}$ relation of Karim et al. (2011) $\left(\operatorname{SFR}\left(M_{\mathrm{h}}\right)=f\left(h\left(M_{\mathrm{h}}\right)\right)\right.$ with $f$ taken from the Karim et al. (2011) fit and $h$ from the results presented in Sect. 4.1). In these two cases, we find a very strong evolution of the ratio between the SFR and the halo mass with redshift ( 1.2 order of magnitude between $z=0$ and $z=2.25$ ). We also see an increase of the halo mass where the instantaneous star formation is the most efficient, from $M_{\mathrm{h}}=3 \times 10^{11} M_{\odot}$ at $z=0$ to $1 \times 10^{12} M_{\odot}$ at $z=2.25$. This evolution is even more pronounced with the second method. The relationship between $M_{\mathrm{h}}$ and SFR $/ M_{\mathrm{h}}$ is close to a log-normal distribution with a typical width $\sim 0.75$ ( $\sim 0.65$ if we use the second method), but presents an excess at high mass $\left(M_{\mathrm{h}}>10^{13} M_{\odot}\right)$. This differs notably from the SFR- $M_{\mathrm{h}}$ relation found by Conroy \& Wechsler (2009) at high mass $\left(M_{\mathrm{h}}>2 \times 10^{12} M_{\odot}\right)$. However, at these high masses the conversion between their stellar mass growth and SFR is less

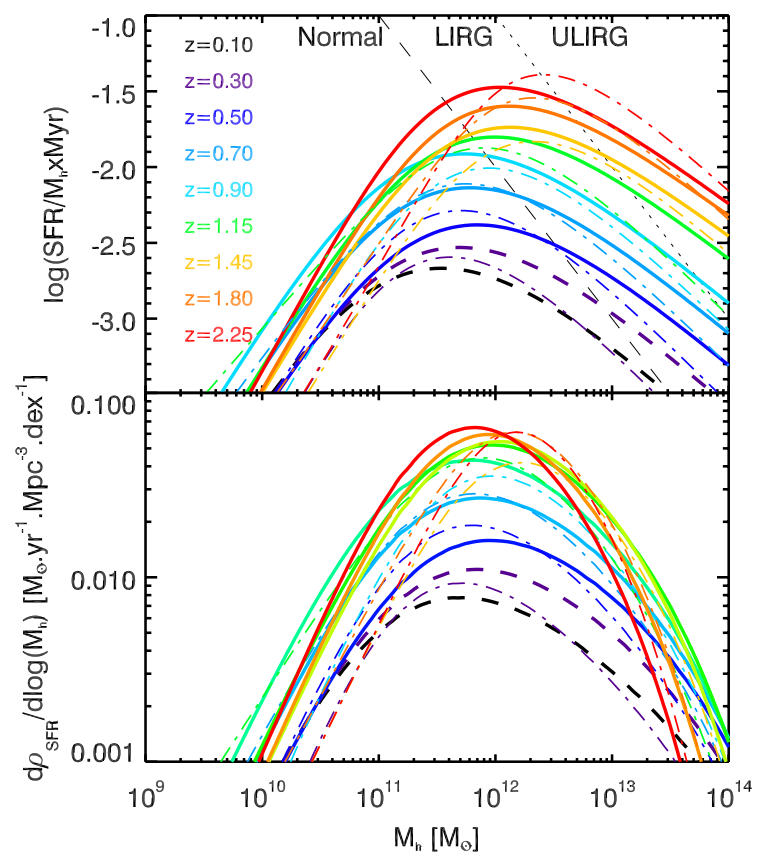

Fig. 3. Upper panel: ratio between SFR and halo mass as a function of halo mass at various redshifts. Solid lines: results from a direct abundance matching of the LFIR and the HMF (we use dashed lines at $z \leq 0.3$ where neglecting the quiescent galaxies has a significant impact, see Sect. 5). Dotted-dashed lines: results from the abundance matching between the SMF and the HMF, combined with the best-fit of the SSFR- $M_{\star}$ relation by Karim et al. (2011). Thin-dashed and dotted lines are the limits between the normal and LIRG, and the LIRG and ULIRG regime, respectively. Lower panel: contribution of the various halo masses to the SFR density.

reliable (see their Sect. 3.2). In contrast to Conroy \& Wechsler (2009) we made no assumption regarding the relation between star formation and halo growth.

From the SFR- $M_{\mathrm{h}}$ relation, we can easily derive the differential contribution of each halo mass to the global SFR density $\rho_{\mathrm{SFR}}$ :

$\frac{\mathrm{d} \rho_{\mathrm{SFR}}}{\mathrm{d} \log \left(M_{\mathrm{h}}\right)}=\frac{\mathrm{d}^{2} N}{\mathrm{~d} M_{\mathrm{h}} \mathrm{d} V} \times \operatorname{SFR}\left(M_{\mathrm{h}}\right)$,

where $\mathrm{d}^{2} N /\left(\mathrm{d} M_{\mathrm{h}} \mathrm{d} V\right)$ is the HMF and $\operatorname{SFR}\left(M_{\mathrm{h}}\right)$ is the SFR associated to a given halo mass provided by the abundance matching. Figure 3 shows the evolution of this observable with halo mass and redshift. We find that the SFR density is dominated by halo masses close to $\sim 7 \times 10^{11} M_{\odot}$, in agreement with the analysis of Conroy \& Wechsler (2009) (their Fig. 10). The distribution is quite sharp with a rapid decrease at smaller and higher mass. We see specifically from this figure that the bulk of star formation at $z \leq 2.3$ never occurs in small systems.

\section{Discussion}

We presented a first application of the abundance matching technique to current infrared data. Our exercise turned out to be surprisingly elucidating. Despite its simplicity, this technique provides a reasonable picture of the evolution of the SFR with the stellar mass, in qualitative agreement with the multi-wavelength measurements at $z>0.3$ (Sect. 4.2). It confirms the strong link between these two quantities. The success of this analysis relies on the hypothesis that the contribution of main-sequence outsider objects to the IR energy output is negligible. Matching 
the LFIR and the SMF including the starburst contribution into LFIR leads to a very strong increase of the SSFR- $M_{\star}$ relation for $M_{\star}>10^{11} M_{\odot}$, which is not observed. Similarly, it artificially increases the $\mathrm{SSFR}-M_{\mathrm{h}}$ relation for $M_{\mathrm{h}}>3 \times 10^{12} M_{\odot}$. The tightness of the sSFR- $M_{\star}$ relation indicates that the SFR is not driven by merger-induced starbursts but instead by a continuous mass-dependent process that is gradually declining with time. Reproducing the evolution of this relation is a challenge for models of galaxy formation (e.g. Davé 2008; Damen et al. 2009).

Our analysis neglects the presence of quiescent massive galaxies at high mass. By comparing the matched abundances with or without quiescent galaxies in the SMF using the Ilbert et al. (2010) measurements, we found that their contribution is negligible $(\leq 0.2$ dex $)$ for $z \geq 0.3$, but reach 0.25 dex at $z=0.3$. A smaller effect $(\sim 0.15 \mathrm{dex})$ at low $z$ is found using the measurements of van der Wel et al. (2010). Because the CIB is dominated by $z \geq 0.3$ galaxies, neglecting the contribution of quiescent galaxies has little impact on its modeling.

Figure 3 demonstrates that the halo mass with the most efficient star formation does not evolve strongly with redshift, with only a decrease of only a factor $\sim 3$ since $z \simeq 2.3$. This may contradict with one meaning of the downsizing phenomenon, which implies which stars are being formed in preferentially smaller systems at later times. This lack of evidence has also been observed by Conroy \& Wechsler (2009) up to $z \sim 1$. This effect could be produced by a poor estimate of the faint-end slope of the LFIR. To investigate this, we repeated our analysis using higher slopes (up to $\alpha=1.8$ ) and found that our conclusion still holds.

The existence of a characteristic mass-scale at high-mass $\left(M_{\mathrm{h}} \simeq 10^{12} M_{\odot}\right)$ has been known since the late 1970s and early 1980s (e.g. Rees \& Ostriker 1977; Silk 1977; White \& Rees 1978). More recently, cosmological simulations also suggest the existence of a critical halo mass, with an important role in shaping up the main properties of galaxy. Below this mass, galaxies are built by cold flows associated with efficient star formation. Above this mass, cooling and star formation are shut down by shock-heating triggered feedback (e.g. Dekel \& Birnboim 2006). Our abundance matching naturally finds this high-mass cutoff for the star-formation (although it is quite a smooth transition). At the low-mass end, there is also strong evidence for a drop in the efficiency of galaxy formation (e.g. Shankar et al. 2006; van den Bosch et al. 2007; Baldry et al. 2008; Kravtsov 2010; Moster et al. 2010; Guo et al. 2010; Bouché et al. 2010). With a very crude approximation of a suppression of accretion and SFR in halo with $M_{\mathrm{h}}<10^{11} M_{\odot}$, Bouché et al. (2010) succeeded in reproducing the mass and redshift dependences of the SFR- $M_{\star}$ and Tully-Fisher relations from $z \sim 2$ to the present. The exact physical processes leading to this mass floor are still under debate. Again, with our simple abundance matching approach, the drop of SFR in low-mass halos appears naturally. These characteristic mass-scales also roughly agree with those required by the constraints coming from the clustering modeling of star forming galaxies (e.g. Cooray et al. 2010; Planck Collaboration 2011) although the detailed relation between our characteristic masses and theirs require additional investigations.

Because the halo mass is the prime quantity that defines the matter clustering on large scales, the link between SFR and halo mass is not only fundamental for understanding the evolution of the galaxies, but also the CIB anisotropies. The current CIB anisotropy models, based on halo occupation distribution prescriptions (Pénin et al. 2012; Planck Collaboration 2011; Amblard et al. 2011), do not take into account that the brighter infrared sources tend to be in more massive halos (although Shang et al. 2011 attempted to address this). Our analysis showed that the relation between SFR and the halo mass has a fairly constant shape from $z=0$ to $z=2$, but significantly shifts with the redshift, the characteristic halo mass varying by a factor $\sim 3$ with redshift (see Fig. 3). With improved models of CIB anisotropies, we can hope to accurately constrain this relation and its evolution with halo mass and redshift. This modeling may be able to decrease the tension between wavelengths discussed in the Planck Collaboration (2011).

Acknowledgements. We thank Andrew Wetzel, whose questions stimulated this work, Mark Sargent, Emanuele Daddi and Lingyu Wang for their insightful comments, Gil Holder for his special perspective. M.B. acknowledge financial support from ERC-StG grant UPGAL 240039. Part of this research was carried out at JPL/Caltech under a contract from NASA.

\section{References}

Amblard, A., Cooray, A., Serra, P., et al. 2011, Nature, 470, 510

Baldry, I. K., Glazebrook, K., \& Driver, S. P. 2008, MNRAS, 388, 945

Bauer, A. E., Conselice, C. J., Pérez-González, P. G., et al. 2011, MNRAS, 417, 289

Behroozi, P. S., Conroy, C., \& Wechsler, R. H. 2010, ApJ, 717, 379

Bouché, N., Dekel, A., Genzel, R., et al. 2010, ApJ, 718, 1001

Caputi, K. I., Cirasuolo, M., Dunlop, J. S., et al. 2011, MNRAS, 413, 162

Chabrier, G. 2003, PASP, 115, 763

Conroy, C., \& Wechsler, R. H. 2009, ApJ, 696, 620

Conroy, C., Prada, F., Newman, J. A., et al. 2007, ApJ, 654, 153

Cooray, A., Amblard, A., Wang, L., et al. 2010, A\&A, 518, L22

Damen, M., Labbé, I., Franx, M., et al. 2009, ApJ, 690, 937

Davé, R. 2008, MNRAS, 385, 147

Dekel, A., \& Birnboim, Y. 2006, MNRAS, 368, 2

Elbaz, D., Dickinson, M., Hwang, H. S., et al. 2011, A\&A, 533, A119

Guo, Q., White, S., Li, C., \& Boylan-Kolchin, M. 2010, MNRAS, 404, 111

Ilbert, O., Salvato, M., Le Floc'h, E., et al. 2010, ApJ, 709, 644

Kajisawa, M., Ichikawa, T., Tanaka, I., et al. 2009, ApJ, 702, 1393

Karim, A., Schinnerer, E., Martínez-Sansigre, A., et al. 2011, ApJ, 730, 61

Kennicutt, R. C. Jr. 1998, ApJ, 498, 541

Kravtsov, A. 2010, Adv. Astron., 2010

Larson, D., Dunkley, J., Hinshaw, G., et al. 2011, ApJS, 192, 16

Magnelli, B., Elbaz, D., Chary, R. R., et al. 2011, A\&A, 528, A35

Mandelbaum, R., Seljak, U., Kauffmann, G., Hirata, C. M., \& Brinkmann, J.

2006, MNRAS, 368, 715

More, S., van den Bosch, F. C., \& Cacciato, M. 2009, MNRAS, 392, 917

Moster, B. P., Somerville, R. S., Maulbetsch, C., et al. 2010, ApJ, 710, 903

Oliver, S., Frost, M., Farrah, D., et al. 2010, MNRAS, 405, 2279

Pénin, A., Doré, O., Lagache, G., \& Béthermin, M. 2012, A\&A, in press, DOI: $10.1051 / 0004-6361 / 201117489$

Pérez-González, P. G., Rieke, G. H., Villar, V., et al. 2008, ApJ, 675, 234

Planck Collaboration 2011, A\&A, 536, A18

Rees, M. J., \& Ostriker, J. P. 1977, MNRAS, 179, 541

Reyes, R., Mandelbaum, R., Gunn, J. E., et al. 2011, MNRAS, submitted [arXiv: 1110.4107$]$

Rodighiero, G., Cimatti, A., Gruppioni, C., et al. 2010, A\&A, 518, L25

Rodighiero, G., Daddi, E., Baronchelli, I., et al. 2011, ApJ, 739, L40

Salpeter, E. E. 1955, ApJ, 121, 161

Sargent, M., Bethermin, M., Daddi, E., \& Elbaz, D. 2011, ApJ, submitted

Saunders, W., Rowan-Robinson, M., Lawrence, A., et al. 1990, MNRAS, 242, 318

Schechter, P. 1976, ApJ, 203, 297

Shang, C., Haiman, Z., Knox, L., \& Oh, S. P. 2011, MNRAS, submitted [arXiv: 1109.1522]

Shankar, F., Lapi, A., Salucci, P., De Zotti, G., \& Danese, L. 2006, ApJ, 643, 14 Silk, J. 1977, ApJ, 211, 638

Tinker, J. L., \& Wetzel, A. R. 2010, ApJ, 719, 88

Tinker, J., Kravtsov, A. V., Klypin, A., et al. 2008, ApJ, 688, 709

Vale, A., \& Ostriker, J. P. 2004, MNRAS, 353, 189

van den Bosch, F. C., Yang, X., Mo, H. J., et al. 2007, MNRAS, 376, 841

van der Wel, A., Bell, E. F., Holden, B. P., Skibba, R. A., \& Rix, H.-W. 2010, ApJ, 714, 1779

White, S. D. M., \& Rees, M. J. 1978, MNRAS, 183, 341

Wilkins, S. M., Trentham, N., \& Hopkins, A. M. 2008, MNRAS, 385, 687

Yun, M. S., Reddy, N. A., \& Condon, J. J. 2001, ApJ, 554, 803 